\title{
Effects of percutaneous transluminal angioplasty of superficial femoral artery on photoplethysmographic pulse transit times
}

\author{
Mikko Peltokangas, Velipekka Suominen, Damir Vakhitov, Janne Korhonen, Jarmo Verho, Ville M. Mattila, Pekka \\ Romsi, Jukka Lekkala, Antti Vehkaoja, and Niku Oksala
}

\begin{abstract}
We analyze the changes in upper and lower limb pulse transit times (PTT) caused by peripheral artery disease (PAD) and its treatment with percutaneous transluminal angioplasty (PTA) of the superficial femoral artery. PTTs were extracted from the photoplethysmograms (PPG) recorded from an index finger and 2nd toes. PTTs were defined between the R-peaks of the ECG and different reference points of the PPG: foot and peak points, maxima of 1 st and 2 nd derivative, and by means of intersecting tangents method. Also the PTTs between the toe and finger pulses were analyzed. Our sample consists of 24 subjects examined before and after the PTA and in 1month follow-up visit. Also 28 older than 65 years controls having normal ankle-to-brachial pressure index (ABI) and no history in cardiovascular diseases as well as 21 younger subjects were examined. The differences between the groups and pre- and posttreatment phases were analyzed by means of non-parametric statistical tests. The changes in the PTTs of upper limb and non-treated lower limb were negligible. The agreement with the reference values, $\mathrm{ABI}$ and toe pressures, was studied by kappaanalysis, resulting in kappa-values of 0.33-0.91. Differences in PTTs were found between pre-treatment state of the treated limb, post-treatment state and the follow-up visit, as well as between the pre-treatment state and controls. If patients' age and systolic blood pressure were taken into consideration, the method of lower limb PTT calculation from the peak point turns out feasible in finding the markers of PAD and monitoring posttreatment vascular remodellation.
\end{abstract}

Index Terms-Atherosclerosis, Photoplethysmography, Peripheral artery disease, Pulse wave measurements.

\section{INTRODUCTION}

Peripheral artery disease (PAD) is a specific form of atherosclerosis affecting mainly the lower limbs, causing stiffening, stenoses or occlusion of the arteries. PAD can be

Manuscript received on November 27, 2017.

M. Peltokangas, J. Verho, A. Vehkaoja, and J. Lekkala are with BioMediTech Institute and Faculty of Biomedical Sciences and Engineering, Tampere University of Technology, Tampere, Finland. Email addresses are firstname.lastname@tut.fi

V. Suominen and D. Vakhitov are with Division of Vascular Surgery, Department of Surgery, Tampere University Hospital, Tampere, Finland.

J. Korhonen is with Division of Interventional Radiology, Department of Radiology, Tampere University Hospital, Tampere, Finland

V. Mattila is with Department of Surgery, Tampere University Hospital, Tampere, Finland, and Surgery, Faculty of Medicine and Life Sciences, University of Tampere, Tampere, Finland.

P. Romsi is with Oulu University Hospital, Oulu, Finland

N. Oksala is with Division of Vascular Surgery, Department of Surgery, Tampere University Hospital, Tampere, Finland, and Finnish Cardiovascular Research Center Tampere, Surgery, Faculty of Medicine and Life Sciences, University of Tampere, Tampere, Finland. asymptomatic for a long time or cause intermittent claudication, critical limb ischemia with rest pain, tissue defects, and result in lower limb amputation. In addition to direct effects, PAD is considered a significant risk factor for severe cardiovascular events such as ischemic stroke or myocardial infarction. Percutaneous transluminal angioplasty (PTA) is a common minimally invasive endovascular treatment in which a stenosed artery is dilated. In most cases, the PTA initiates histological remodellation of the artery, but in some cases induces the development of restenosis which results in the recurrence of the symptoms. For this reason, the patients are subjected to a 1-month follow-up period in order to ensure the technical success of the PTA.

Current diagnostic methods for PAD include expensive imaging techniques (magnetic resonance angiography (MRA), $\mathrm{X}$-ray contrast-agent angiography, or computed tomography angiography (CTA)). More cost-effective alternatives applicable mostly for screening purposes include ankle-to-brachial pressure index (ABI) with highly varying sensitivity and specificity [1], toe-to-brachial pressure index (TBI) and systolic toe pressures, but their reliability is limited especially with diabetic or mediasclerotic patients [2], [3].

Earlier studies have shown that blood pressure, height, arterial stiffening as a result of aging, and the existence of PAD affect the pulse transit times (PTT) [4]-[8]. We hypothesized that the PTA of superficial femoral artery (SFA) affects especially the lower-limb PTTs. In this study, we studied how PTA of the SFA changes PTTs and how the upper and lower limb PTTs of PAD patients differ from the PTTs of same-aged healthy subjects and how the values depend on the age. We extracted PTTs between the R-peak of the ECG and different reference points of the pulse waves (PW) as well as between reference points extracted from the PWs recorded from the index finger and 2nd toe. In both situations, the PTTs were extracted for both immediate pre- and post-treatment situations as well as for the situation in the follow-up visit.

\section{MATERIALS AND METHODS}

\section{A. Study subjects}

The data was collected in two separate clinical studies (S1 and S2). The PW-signals of study S1 were recorded from 27 patients undergoing PTA of the SFA. Three of them were excluded from the analysis for the following reasons: 1) the lack of data from follow-up visit, 2) a patient was found to be 
Table I

DATA DESCRIBING THE STUDY SUBJECT POPULATION. THE VALUES ARE PRESENTED AS MEDIAN (1ST QUARTILE-3RD QUARTILE)

\begin{tabular}{|c|c|c|c|c|}
\hline Group & $\begin{array}{c}(n=24) \\
\text { (PTA-patients) }\end{array}$ & $\begin{array}{l}\mathrm{C}(n=28) \\
\text { (Control) }\end{array}$ & $\begin{array}{c}\mathrm{M}(n=9) \\
\text { (Middle-aged) }\end{array}$ & $\begin{array}{l}\mathrm{Y}(n=12) \\
\text { (Young) }\end{array}$ \\
\hline Age (years) & $72(68-76)$ & $72(68-77)$ & $56(50-61)$ & $30(26-34)$ \\
\hline \multicolumn{2}{|c|}{ Treated lower limbs: 26} & \multirow{6}{*}{$\begin{array}{c}1.10 \\
(1.06-1.22)\end{array}$} & - & - \\
\hline ABI, pre-PTA & $0.61(0.50-0.75)$ & & - & - \\
\hline post-PTA & $0.96(0.82-1.04)$ & & - & - \\
\hline \multicolumn{2}{|c|}{ Non-treated lower limbs: 22} & & - & - \\
\hline ABI, pre-PTA & $|0.88(0.75-1.02)|$ & & - & - \\
\hline post-PTA & $0.94(0.72-1.08)$ & & - & - \\
\hline \multicolumn{2}{|c|}{ Toe pressure, treated limb: } & - & - & - \\
\hline Pre-PTA (mmHg) & $59(42-86)$ & - & - & - \\
\hline Post-PTA (mmHg) & $95(76-122)$ & - & - & - \\
\hline \multicolumn{2}{|c|}{ Toe pressure, non-treated limb: } & - & - & - \\
\hline Pre-PTA (mmHg) & $76(53-118)$ & - & - & - \\
\hline Post-PTA (mmHg) & $100(66-122)$ & - & - & - \\
\hline Height $(\mathrm{cm})$ & $170(166-176)$ & $170(165-180)$ & $180(175-182)$ & $179(176-183)$ \\
\hline SBP $(\mathrm{mmHg})$ & $169(151-182)$ & $144(135-163)$ & $136(131-151)$ & $144(136-154)$ \\
\hline DBP $(\mathrm{mmHg})$ & $78(72-83)$ & $83(78-91)$ & $86(66-92)$ & $80(72-89)$ \\
\hline Men & $16(66.7 \%)$ & $9(29.0 \%)$ & $9(100 \%)$ & $12(100 \%)$ \\
\hline Diabetes & $11(45.8 \%)$ & $1(3.2 \%)$ & $1(11.1 \%)$ & $1(8.3 \%)$ \\
\hline Dyslipidemia & $22(91.7 \%)$ & $5(16.1 \%)$ & $0(0 \%)$ & $0(0 \%)$ \\
\hline Rheumatoid a. & $3(12.5 \%)$ & $5(16.1 \%)$ & $0(0 \%)$ & $1(8.3 \%)$ \\
\hline History of smoking & $11(45.8 \%)$ & $7(22.6 \%)$ & $0(0 \%)$ & $0(0 \%)$ \\
\hline
\end{tabular}

$\mathrm{ABI}=$ ankle-to-brachial pressure index; $\mathrm{SBP}=$ systolic blood pressure; DBP = diastolic blood pressure; Rheumatoid a. = Rheumatoid arthritis

unsuitable for PTA during the imaging, and 3) extremely low peripheral perfusion preventing reliable determination of PTT. In study S1, 14 left and 12 right lower limbs were treated, including two patients with bilateral treatment. More detailed data on the study subjects is shown in Table I. The inclusion criteria of study $\mathrm{S} 1$ were abnormal $\mathrm{ABI}$ reading $(\mathrm{ABI}<0.9$ or $\mathrm{ABI}>1.3$ ), relevant symptoms, diagnosed stenosis in SFA as a result of MRA and a patient considered as a candidate for SFA PTA. A pacemaker was considered as exclusion criterion. A majority of the study subjects in study S1 met the criterion $\mathrm{ABI}<0.9$. Only one of the included patients had pre-treatment ABI higher than 1.3 which indicates incompressible arteries in a lower limb. 15 non-treated lower limbs out of $22(68 \%)$ also had the $\mathrm{ABI}$ value outside the normal range of $0.9<\mathrm{ABI}<1.3$ at least in one of the pre- or post-treatment ABI measurements.

The control subjects for the study were recruited and data recorded as a part of study $\mathrm{S} 2$. The sample consisted of 31 at least 65-year-old subjects (group $\mathrm{C}$ ) having normal ABI and no history in cardiovascular diseases. Three of these subjects were excluded from the study due to miscellaneous reasons (technical problems or subject's condition did not allow the placement of a sensor to a particular measurement point.) The remaining 21 control subjects (groups $\mathrm{M}$ and $\mathrm{Y}$ ) consisted of younger control subjects (22-63 years) having no diagnosed cardiovascular diseases.

\section{B. Reference values}

Both $\mathrm{ABI}$ and toe pressure were utilized as reference values. Generally, the ABI is defined as a ratio of the systolic blood pressures in the ankle and arm, i.e. $\mathrm{ABI}=P_{\text {ankle }} / P_{\text {brachial }}$. In this study, ABI was computed by dividing the highest ankle pressure (either ADP (arteria dorsalis pedis) or ATP (arteria tibialis posterior)) by the highest arm pressure (left or right). Toe pressures refer to the systolic pressures measured from the hallux. Both reference values were collected by using an automated measurement device, Falcon Pro (Viasonix, Israel). Pre-treatment (visit at outpatient clinics before the PTA) and follow-up visit $\mathrm{ABI}$ and toe pressure measurements were conducted as a part of standard clinical protocol and practice.

\section{Ethics and patient safety}

The patient measurements of study S1 were approved by the local ethical review board of Pirkanmaa hospital district (R15107) and the Finnish National Supervisory Authority of Health and Welfare (Valvira, ID 309). The study was registered to ClinicalTrials.gov (ID: NCT02725307). The patient measurements of study S2 were approved by the local ethical review board of the hospital district (R14096) and Valvira (ID 272).

\section{Measurement hardware}

The measurement data was collected using transmission mode PPG probes having a LED wavelength of $905 \mathrm{~nm}$. Bipolar ECG was measured using conventional disposable $\mathrm{Ag} / \mathrm{AgCl}$ electrodes placed under the clavicles. Both types of sensors were connected into a synchronous wireless body sensor network [9]. The sampling frequency was $250 \mathrm{~Hz}$ for the ECG signals and $500 \mathrm{~Hz}$ for the PPG signals. The ECG signals were interpolated to have a sampling frequency of $500 \mathrm{~Hz}$ in further signal analysis by implementing a low-pass interpolation algorithm [10].

\section{E. Sensor placement}

In study $\mathrm{S} 1$, PPG probes were placed on index finger and both second toes for recording blood volume PW signals. The test subjects were in supine position during the measurements. The measurements were conducted during pre-, peri-, and immediate post-treatment phases of the PTA and during the follow-up visit within approximately one month (average 33.5 days, standard deviation 4.1 days). The sensors were placed before the normal preparation of the PTA and were removed within 5-10 minutes after the PTA. In study S2, the sensors were placed as in study S1, but the signals from the lower limb were recorded and analyzed unilaterally. The duration of the measurement was approximately 15 minutes in study S2. In both studies, the PTTs averaged over the whole recording or particular phase of the PTA were used in the analysis.

\section{F. Signal pre-processing}

The PW-signals were pre-processed as in [11]. The signals were filtered with a Savitzky-Golay (SG) smoothing filter having a window length of 91 samples $(182 \mathrm{~ms})$ and a polynomial order of 2 . In addition, the signals were lowpassfiltered with a finite impulse response filter having a cut-offfrequency of $10 \mathrm{~Hz}$, transition band of $10 \mathrm{~Hz}-12 \mathrm{~Hz}$, pass-band ripple of $0.05 \mathrm{~dB}$ and stop-band attenuation of $100 \mathrm{~dB}$ [12] using forward-backward filtering. 


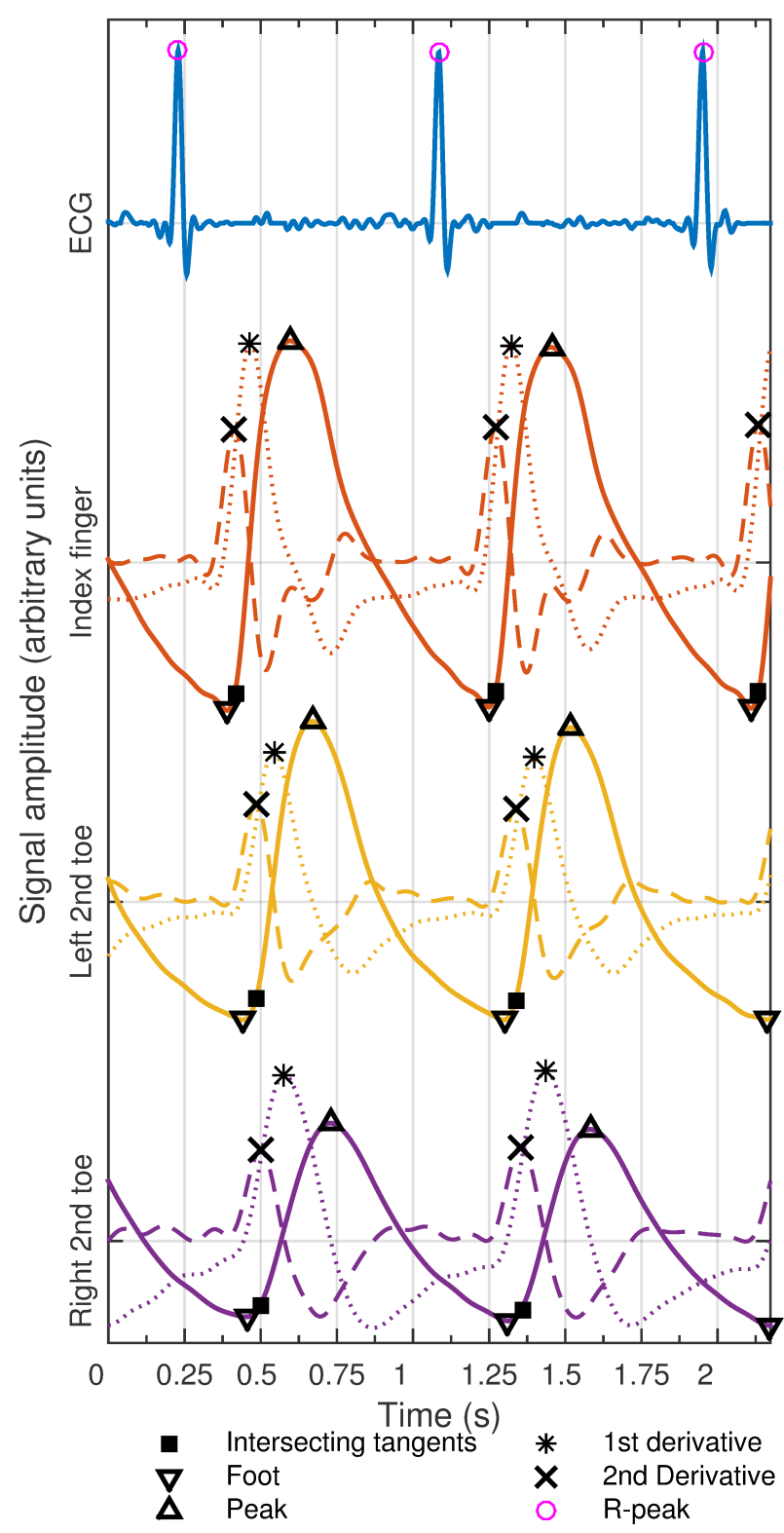

Figure 1. Examples of the timings of the pulse waves and the R-peaks of the low-pass-filtered baseline-removed ECG for pre-treatment situation of 70year-old female whose right lower limb will be treated with PTA.

\section{G. Pulse transit times}

PTT and pulse wave velocity (PWV) extracted from upper limb PWs are commonly studied to evaluate on arterial aging and stiffness [5], [13]. In this study, we concentrated on the lower limb PTT analysis in order to find the effects of PAD and the effects of minimally invasive treatment, i.e. PTA of the SFA.

The resistance and compliance of the arterial pathway affect the PW propagation and reflection [5], [7], or actually the frequency response of the arterial tree. We assume that in a lower limb with PAD lesions, the increased flow resistance due to stenoses causes a low-pass filter-type response: the observed peripheral PW is delayed compared with young and healthy arteries and its higher-frequency features are dampened which is seen as a rounded peak and a loss of dicrotic notch. Also the blood pressure in the diseased lower limb is decreased due to stenoses, causing lower PWV in the lower limb.

Two types of PTTs were extracted, 1) ECG-PTTs and 2) toe-finger-PTTs. The ECG-PTTs $\left(\mathrm{PTT}_{\mathrm{E}}\right)$ were calculated between the time of the R-peak $t_{\mathrm{R}}$ of the ECG and the time of a reference point $t_{\text {ref }}$ in PPG as

$$
\mathrm{PTT}_{\mathrm{E}}=t_{\mathrm{ref}}-t_{\mathrm{R}}
$$

The toe-finger PTTs $\left(\mathrm{PTT}_{\mathrm{TF}}\right)$ were computed as

$$
\mathrm{PTT}_{\mathrm{TF}}=\mathrm{PTT}_{\text {toe }}-\mathrm{PTT}_{\text {finger }}
$$

in which $\mathrm{PTT}_{\text {toe }}$ and $\mathrm{PTT}_{\text {finger }}$ are the ECG-PTTs for 2nd toe PPG and index finger PPG.

We initially evaluated several well-established reference points in PPG. These points (Fig. 1) were foot point, the maximum of the 1 st derivative, the maximum of the 2 nd derivative and a point found by intersecting tangents method [13]. In addition to these well-established reference points, we extracted PTTs having a peak point of a PW as a reference point since the peak-point based lower limb PTT has been reported to indicate PAD lesions [7], [8]. Besides the ECGPTTs, toe-finger PTTs between the reference points of the index finger and the reference points of 2 nd toes were studied.

A foot point was defined as the minimum between two successive PWs, and peak point as the maximum of the PW (Fig. 1). The 1st and 2nd derivatives were approximated as discrete differences, and reference points based on them were defined as the maxima found between the foot point and the peak point (Fig. 1). A reference point for intersecting tangent is the intersection of horizontal line through the foot point and the line fitted to the points around the steepest slope of the rising edge of the PW. The line was fitted to the maximum number of points in a such way that the correlation remained greater or equal than 0.999 between the fitted line and the points of the PW [13].

The PTTs were compared between the treated lower limb and control groups. In addition, differences $\Delta \mathrm{PTT}_{\mathrm{ab}}$ and $\Delta \mathrm{PTT}_{\mathrm{fb}}$ were computed to assess the effect of the treatment and post-treatment vascular remodellation on the PTTs. $\triangle \mathrm{PTT}_{\mathrm{ab}}$ was defined between the pre-operative PTTs $\left(\mathrm{PTT}_{\mathrm{b}}, \mathrm{b}=\right.$ before $)$ and immediate post-treatment PTTs $\left(\mathrm{PTT}_{\mathrm{a}}, \mathrm{a}=\mathrm{after}\right)$ as

$$
\Delta \mathrm{PTT}_{\mathrm{ab}}=\mathrm{PTT}_{\mathrm{a}}-\mathrm{PTT}_{\mathrm{b}}
$$

and $\Delta \mathrm{PTT}_{\mathrm{fb}}$ between $\mathrm{PTT}_{\mathrm{b}}$ and PTTs at follow-up visit $\left(\mathrm{PTT}_{\mathrm{f}}\right)$ as

$$
\Delta \mathrm{PTT}_{\mathrm{fb}}=\mathrm{PTT}_{\mathrm{f}}-\mathrm{PTT}_{\mathrm{b}}
$$

for both treated and non-treated lower limbs as well as for the upper limbs. Labels used for different PTT measures are presented in Table II.

We did not analyze or compare directly the PTT values between treated and non-treated limbs since $68 \%$ of the reference values (ABI readings) of the non-treated limb were outside the normal range at least in one of the two measurements, indicating that many subject had PAD lesions also in the healthier non-treated lower limb. 
Table II

NOMENCLATURE OF DIFFERENT DATASETS AND CALCULATED PTT MEASURES.

\begin{tabular}{|c|c|}
\hline Symbol & Explanation \\
\hline $\mathrm{T}_{\mathrm{b}}$ & $\mathrm{PTT}_{\mathrm{b}}$ for treated lower limb (pre-treatment measurement, $\mathrm{b}=\mathrm{before}$ ) \\
\hline $\mathrm{F}_{\mathrm{b}}$ & $\mathrm{PTT}_{\mathrm{b}}$ for index finger (pre-treatment measurement) \\
\hline $\mathrm{C}$ & Group of control subjects (see Table I) \\
\hline M & Group of middle-aged subjects (see Table I) \\
\hline $\mathrm{Y}$ & Group of young subjects (see Table I) \\
\hline $\mathrm{T}_{\mathrm{ab}}$ & $\begin{array}{l}\Delta \mathrm{PTT}_{\mathrm{ab}} \text { for treated lower limb (difference between immediate post- } \\
\text { and pre-treatment states, } \mathrm{a}=\text { after, } \mathrm{b}=\text { before) }\end{array}$ \\
\hline $\mathrm{T}_{\mathrm{fb}}$ & $\begin{array}{l}\Delta \mathrm{PTT}_{\mathrm{fb}} \text { for treated lower limb (difference between follow-up visit } \\
\text { and pre-treatment state) }\end{array}$ \\
\hline $\mathrm{N}_{\mathrm{ab}}$ & $\begin{array}{l}\Delta \mathrm{PTT}_{\mathrm{ab}} \text { for non-treated lower limb (difference between immediate } \\
\text { post- and pre-treatment states) }\end{array}$ \\
\hline $\mathrm{N}_{\mathrm{fb}}$ & $\begin{array}{l}\Delta \mathrm{PTT}_{\mathrm{fb}} \text { for non-treated lower limb (difference between follow-up } \\
\text { visit and pre-treatment state) }\end{array}$ \\
\hline $\mathrm{F}_{\mathrm{ab}}$ & $\begin{array}{l}\Delta \mathrm{PTT}_{\mathrm{ab}} \text { for finger (difference between immediate post- and pre- } \\
\text { treatment states) }\end{array}$ \\
\hline $\mathrm{F}_{\mathrm{fb}}$ & $\begin{array}{l}\Delta \mathrm{PTT}_{\mathrm{fb}} \text { for finger (difference between follow-up visit and pre- } \\
\text { treatment state) }\end{array}$ \\
\hline
\end{tabular}

\section{H. PTT dependence on age, blood pressure and height}

Earlier studies [4], [5] have indicated that age is the most significant confounding factor in PTTs in healthy subjects, but also the systolic blood pressure and subject's height have an effect on PTTs. For this reason, we utilized multiple linear regression models to study if these confounders explain the differences between different study groups. The models were developed based on the middle-aged (M) and younger (Y) subjects of study S2 (see Table I). We initially included also diastolic blood pressure in our model, but because neither related studies [4], [5] nor our results indicated any dependence on diastolic blood pressure, we left it out.

According to the results in Table III, age and systolic blood pressure are associated with the toe-based ECG-PTTs $\left(\mathrm{PTT}_{\mathrm{E}}\right)$ and peak-point based toe-finger-PTT $\left(\mathrm{PTT}_{\mathrm{TF}}\right)$. Age was the only explanatory variable in the finger-based ECGPTTs and other than peak-point based toe-finger-PTTs. Other studies [4], [5] have reported also the height dependence of the PTT. However, height did not associate $(p>0.05$ and mostly $p>0.50$ ) with any PTTs in groups $\mathrm{M}$ and $\mathrm{Y}$, so the height dependency coefficients were not included in Table III. This may be caused by the limited height distributions of the subjects (Table I).

In case of statistically significant dependence, the age and systolic blood pressure adjustments were implemented for all

Table III

COEFFICIENTS USED IN AGE AND SYSTOLIC BLOOD PRESSURE ADJUSTMENT.

\begin{tabular}{|c|c|c|c|c|c|c|}
\hline \multirow[b]{2}{*}{$\begin{array}{l}\text { Ref. } \\
\text { point }\end{array}$} & \multicolumn{2}{|c|}{ ECG-PTT (toe) } & \multicolumn{2}{|c|}{ ECG-PTT (finger) } & \multicolumn{2}{|c|}{ Finger-toe PTT } \\
\hline & $\begin{array}{l}\text { age }\left(c_{a}\right) \\
\text { ms/year }\end{array}$ & $\begin{array}{l}\mathrm{SBP}\left(c_{s}\right) \\
\mathrm{ms} / \mathrm{mmHg}\end{array}$ & $\begin{array}{c}\text { age }\left(c_{a}\right) \\
\text { ms/year }\end{array}$ & $\begin{array}{l}\mathrm{SBP}\left(c_{s}\right) \\
\mathrm{ms} / \mathrm{mmHg}\end{array}$ & $\begin{array}{l}\text { age }\left(c_{a}\right) \\
\text { ms/year }\end{array}$ & $\begin{array}{l}\mathrm{SBP}\left(c_{s}\right) \\
\mathrm{ms} / \mathrm{mmHg}\end{array}$ \\
\hline Foot & $-1.6^{d}$ & $-0.82^{b}$ & $-1.1^{c}$ & $-0.27^{\mathrm{NS}}$ & $-1.1^{a}$ & $-0.79^{\mathrm{NS}}$ \\
\hline Foot, ref. & $\begin{array}{c}\mathrm{f} . \\
-1.6[5]\end{array}$ & $\begin{array}{c}-0.95[4] \\
-1.0[5]\end{array}$ & $-0.6[5]$ & $-0.4[5]$ & $-0.58[4]$ & $-0.32[4]$ \\
\hline Peak & $-1.2^{a}$ & $-1.0^{a}$ & $1.6^{c}$ & $0.07^{\mathrm{NS}}$ & $-2.7^{d}$ & $-1.2^{b}$ \\
\hline $1 \mathrm{st}$ d. & $-1.6^{d}$ & $-1.0^{c}$ & $-0.85^{a}$ & $-0.36^{\mathrm{NS}}$ & $-1.4^{b}$ & $-1.0^{\mathrm{NS}}$ \\
\hline 2nd d. & $-1.7^{d}$ & $-1.0^{c}$ & $-1.0^{b}$ & $-0.37^{\mathrm{NS}}$ & $-1.3^{a}$ & $-0.97^{\mathrm{NS}}$ \\
\hline Int. tan. & $-1.7^{d}$ & $-0.97^{c}$ & $-1.0^{b}$ & $-0.33^{\mathrm{NS}}$ & $-1.3^{a}$ & $-0.94^{\mathrm{NS}}$ \\
\hline
\end{tabular}

$a: p<0.05 ; b: p<0.025 ; c: p<0.01 ; d: p<0.001$; NS: not significant study groups $\mathrm{T}_{\mathrm{b}}, \mathrm{C}, \mathrm{M}$ and $\mathrm{Y}$ as

$$
\mathrm{PTT}_{c, j}=\mathrm{PTT}_{j}-c_{a} \cdot a_{j}-c_{s} \cdot s_{j}
$$

in which $\mathrm{PTT}_{c, j}$ is the corrected PTT for subject $j, \mathrm{PTT}_{j}$ is the original PTT for subject $j, c_{\mathrm{a}}$ is the age-dependency coefficient, $a_{j}$ is the age of subject $j, c_{\mathrm{s}}$ is the systolic blood pressure dependency coefficient, and $s_{j}$ is the systolic blood pressure of subject $j$. The estimated coefficients $c_{a}$ and $c_{s}$ are shown in Table III.

\section{Statistical methods}

Different study groups were compared by implementing two-tailed non-parametric Mann-Whitney U-tests since the sample sizes were relatively small and the distributions were skewed in part of the cases. The changes at intra-subject level as a result of the PTA were tested by means of twotailed non-parametric sign test for the same reasons as the implementing of Mann-Whitney U-tests. P-values less than 0.05 were considered statistically significant.

\section{J. PTT comparison with reference values}

Fixed free-marginal multirater $\kappa$-analysis [14] was performed in order to study if the PTT-values of the treated lower limb and the reference values (ABI and toe pressure) change in the same direction. Fixed free-marginal multi-rater $\kappa$ was selected because it is not sensitive to the asymmetry of the data. The analysis was performed for both ECG-PTT and toe-finger PTT. Decrease in PTT-value and increase in ABI and toe pressure were considered as a change towards better condition.

\section{RESULTS}

The results related to the maxima of the $1 \mathrm{st}$ and 2 nd derivatives as well as the intersecting tangents had similar trends with age and presence of PAD as the results of the foot point based PTTs in both upper and lower limbs. For this reason, we concentrate on the results of foot and peak point based PTTs which produced different results especially with upper limb PTTs. Further results related to the methods based on the 1 st ans 2 nd derivatives and intersecting tangents are presented as supplementary material.

\section{A. ECG-PTTs}

The distributions of the absolute ECG-PTTs extracted from the 2nd toe PPGs are shown in Fig. 2a-b for foot and peak points for the pre-treatment situation of treated lower limb $\left(\mathrm{T}_{\mathrm{b}}\right)$ and control subjects $(\mathrm{C}, \mathrm{M}$ and $\mathrm{Y})$. The lower limb PTTs for the control group $(\mathrm{C})$ differ significantly $(p=0.0057$ or less) from the pre-treatment PTT-values of the treated limb $\left(\mathrm{T}_{\mathrm{b}}\right)$ independently on the reference point of the PPG. As seen in Fig. 2a-b, the toe-PTTs have a decreasing trend with age, but show an increase with existence of PAD. The upper limb PTTs have similar trend with the age in all the studied reference points except peak point derived PTT (Fig. 3a-b for foot and peak points). In the upper limb PTTs, the differences between the control group (C) and measurements with PAD 

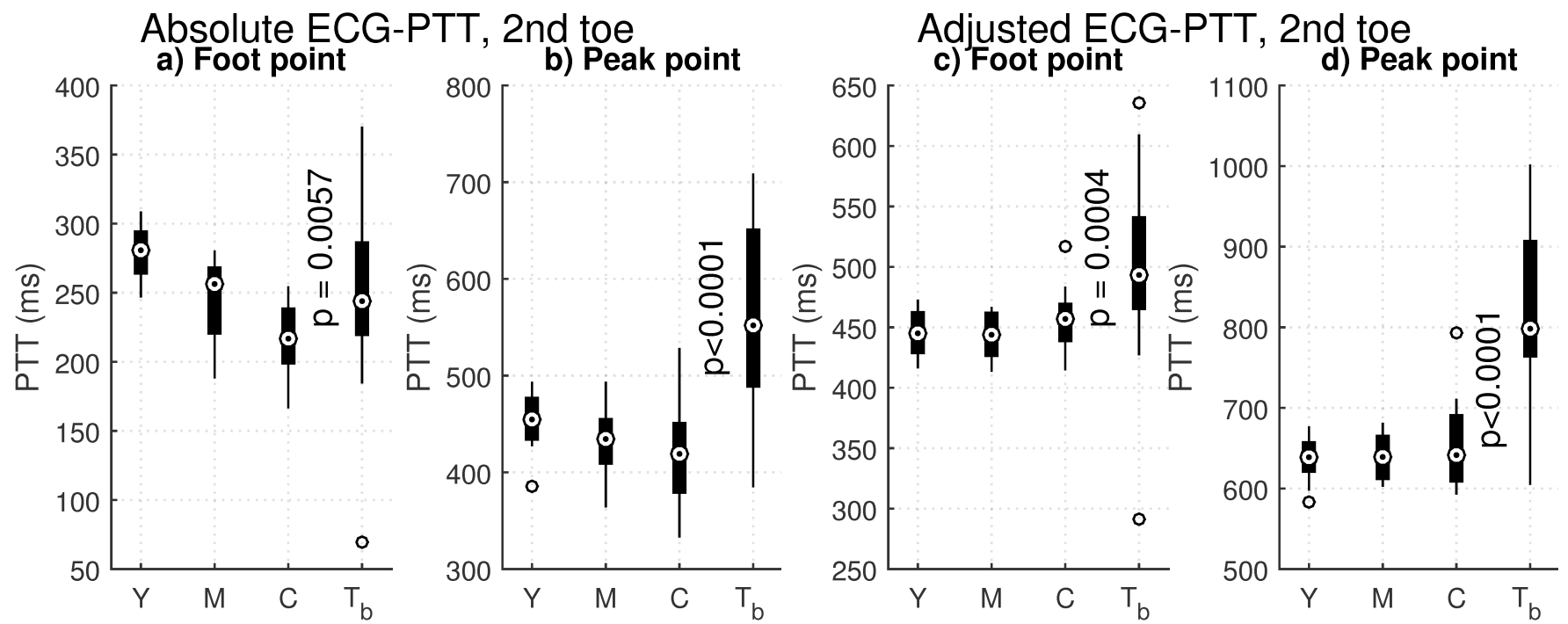

Figure 2. Distributions of the absolute and adjusted PTTs for 2 nd toe. $\mathrm{T}_{\mathrm{b}}=$ treated lower limb before the treatment, $\mathrm{C}=$ at least 65 -year-old control subject, $\mathrm{M}=$ middle-aged subjects, $\mathrm{Y}=$ young subjects. P-values are for 2-tailed Mann-Whitney U-test between $\mathrm{T}_{\mathrm{b}}$ and $\mathrm{C}$.
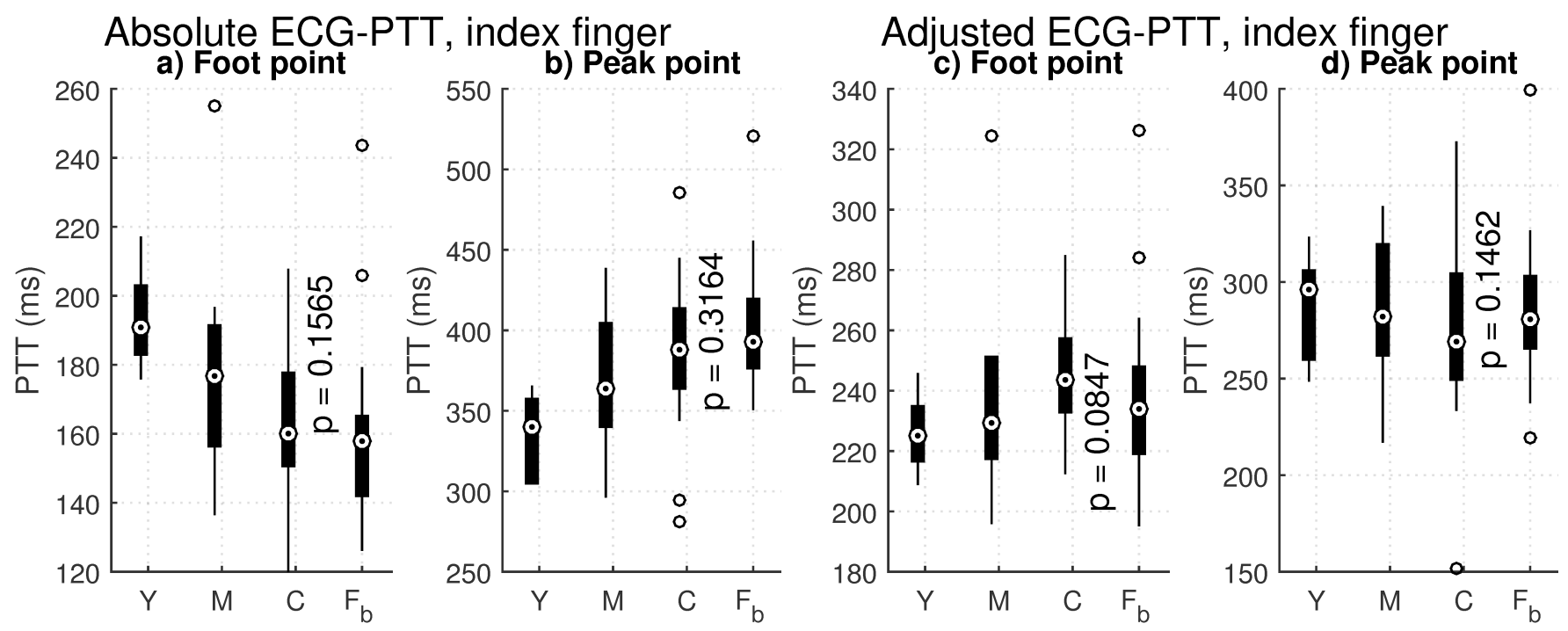

Figure 3. Distributions of the absolute and adjusted PTTs for index finger PPG. $F_{b}=$ PAD patients before the treatment, $C=$ at least 65 -year-old control subjects, $\mathrm{M}=$ middle-aged subjects, $\mathrm{Y}=$ young subjects. $\mathrm{P}$-values are for 2-tailed Mann-Whitney $\mathrm{U}$-test betweeen $\mathrm{F}_{\mathrm{b}}$ and $\mathrm{C}$.

patients (group $\mathrm{F}_{\mathrm{b}}$ ) in Fig. 3a-b) are smaller than in case of the lower limbs $(p=0.156$ or greater). More detailed data including the PTT-distributions of the treated and non-treated lower limbs at different phases are published as supplementary material.

As the PTTs depend on age and systolic blood pressure, we implemented the adjustment presented in Eq. 6 with statistically significant coefficients shown in Table III. The distributions of adjusted PTTs are shown in Figs. 2c-d and $3 \mathrm{c}-\mathrm{d}$ for 2 nd toe and index finger, respectively. The 2 nd toe PTTs for the control group (C) differ significantly $(p=0.0004$ or less) from the pre-treatment PTT-values of the treated limb $\left(\mathrm{T}_{\mathrm{b}}\right)$. With the presented upper limb PTTs, the differences between the control group and the measurements with PAD patients $\left(\mathrm{F}_{\mathrm{b}}\right)$ are less significant $(p>0.05)$.

The distributions of the changes in ECG-PTTs as a result of the SFA PTA are shown in Fig. 4 with the p-values returned by the two-tailed sign tests. The distributions are shown for foot and peak-point based ECG-PTTs of the 2nd toe of the treated $\operatorname{limb}\left(\mathrm{T}_{\mathrm{ab}}\right.$ and $\left.\mathrm{T}_{\mathrm{fb}}\right)$, the 2nd toe of the non-treated limb $\left(\mathrm{N}_{\mathrm{ab}}\right.$ and $\left.\mathrm{N}_{\mathrm{fb}}\right)$, and finger $\left(\mathrm{F}_{\mathrm{ab}}\right.$ and $\left.\mathrm{F}_{\mathrm{fb}}\right)$ in two situations: 1) pre-treatment vs. immediate post-treatment (Eq. (4)), and 2) pre-treatment vs. follow-up visit (Eq. (5)). The difference in the ECG-PTTs for the treated lower limb $\left(\mathrm{T}_{\mathrm{ab}}\right.$ and $\left.\mathrm{T}_{\mathrm{fb}}\right)$ show consistent and statistically significant differences in both cases whereas the differences in ECG-PTTs for non-treated lower $\operatorname{limb}\left(\mathrm{N}_{\mathrm{ab}}\right.$ and $\left.\mathrm{N}_{\mathrm{fb}}\right)$ do not show neither consistent nor statistically significant differences at group level between different measurements. The differences in finger ECG-PTTs $\left(F_{a b}\right.$ and $\left.F_{f b}\right)$ do not show strong differences between different measurements. 
ECG-PTT changes $\Delta$ PTT ${ }_{\text {ab }}$ and $\Delta \mathrm{PTT}_{\mathrm{fb}}$

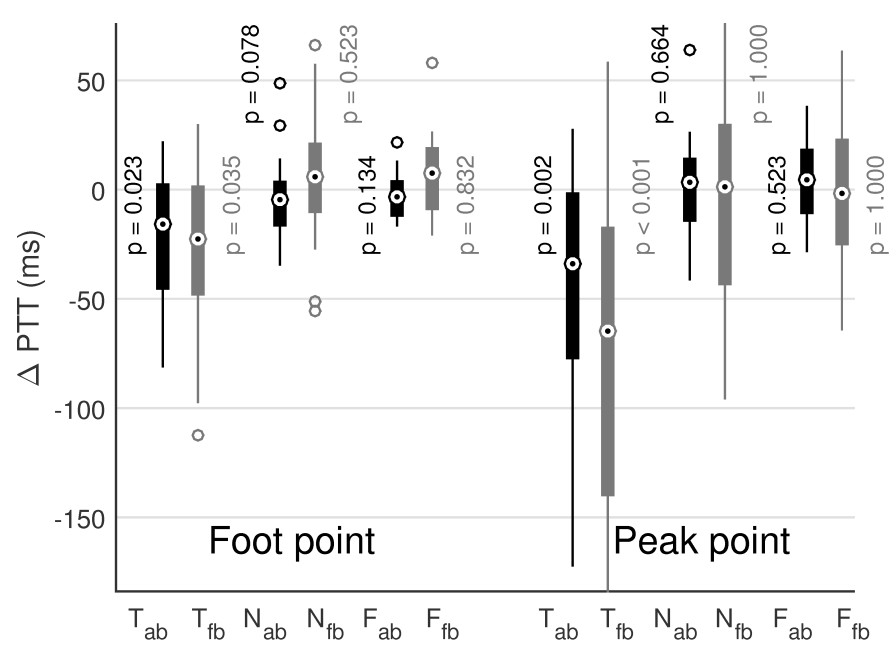

Figure 4. Changes in foot and peak point based PTTs between different recordings. Labels $\mathrm{T}, \mathrm{N}$, and $\mathrm{F}$ refer to treated lower limb, non-treated lower limb and index finger, respectively. Subscript ab refers to difference between pre-treatment $(\mathrm{b}=$ before $)$ and immediate post-treatment $(\mathrm{a}=\mathrm{after})$ conditions, and subscript $\mathrm{fb}$ refers to difference between pre-treatment condition and follow-up visit.

\section{B. Toe-finger PTTs}

The distributions of toe-finger PTTs are shown in Fig. 5 both as absolute values and after age and systolic blood pressure adjustment. Without adjustment, the toe-finger PTTs tended to decrease with age, but increase with the existence of PAD. This is similar behaviour as in the ECG-PTTs of the lower limbs. Both absolute and adjusted toe-finger PTTs of the control group (C) differ significantly $(p<0.0001)$ from the pretreatment toe-finger PTTs of treated lower limb $\left(\mathrm{T}_{\mathrm{b}}\right)$ in case of all the studied reference points. More detailed data including the toe-finger PTT-distributions of non-treated lower limb at different phases are presented in Supplementary material.

Fig. 6 presents the differences of toe-finger PTTs between immediate post- and pre-treatment conditions $\left(\mathrm{T}_{\mathrm{ab}}\right.$ and $\left.\mathrm{N}_{\mathrm{ab}}\right)$ and a situation at follow-up visit and pre-treatment situation $\left(\mathrm{T}_{\mathrm{fb}}\right.$ and $\left.\mathrm{N}_{\mathrm{fb}}\right)$ for both treated limb $(\mathrm{T})$ and non-treated limb (N). The results show that there are significant $(p=0.027$ or less) immediate differences in toe-finger PTTs of the treated limb. The difference between pre-treatment and follow-up conditions is even more distinct $(p<0.001)$. No significant differences were seen in the toe-finger PTTs of non-treated limbs immediately after the treatment nor at the follow-up visit.

\section{Agreement with reference values}

The free-marginal multirater $\kappa$-values are presented in Table IV for the changes in lower-limb PTT-values vs. changes in reference values $A B I$ and toe pressure. Peak point and 1st derivative based ECG-PTTs of lower-limbs have the best agreement between the reference values. In case of toe-finger PTTs, the PTTs based on the 1st derivative and intersecting tangents methods result in the best agreement with the reference values. Especially the toe-finger PTTs have better
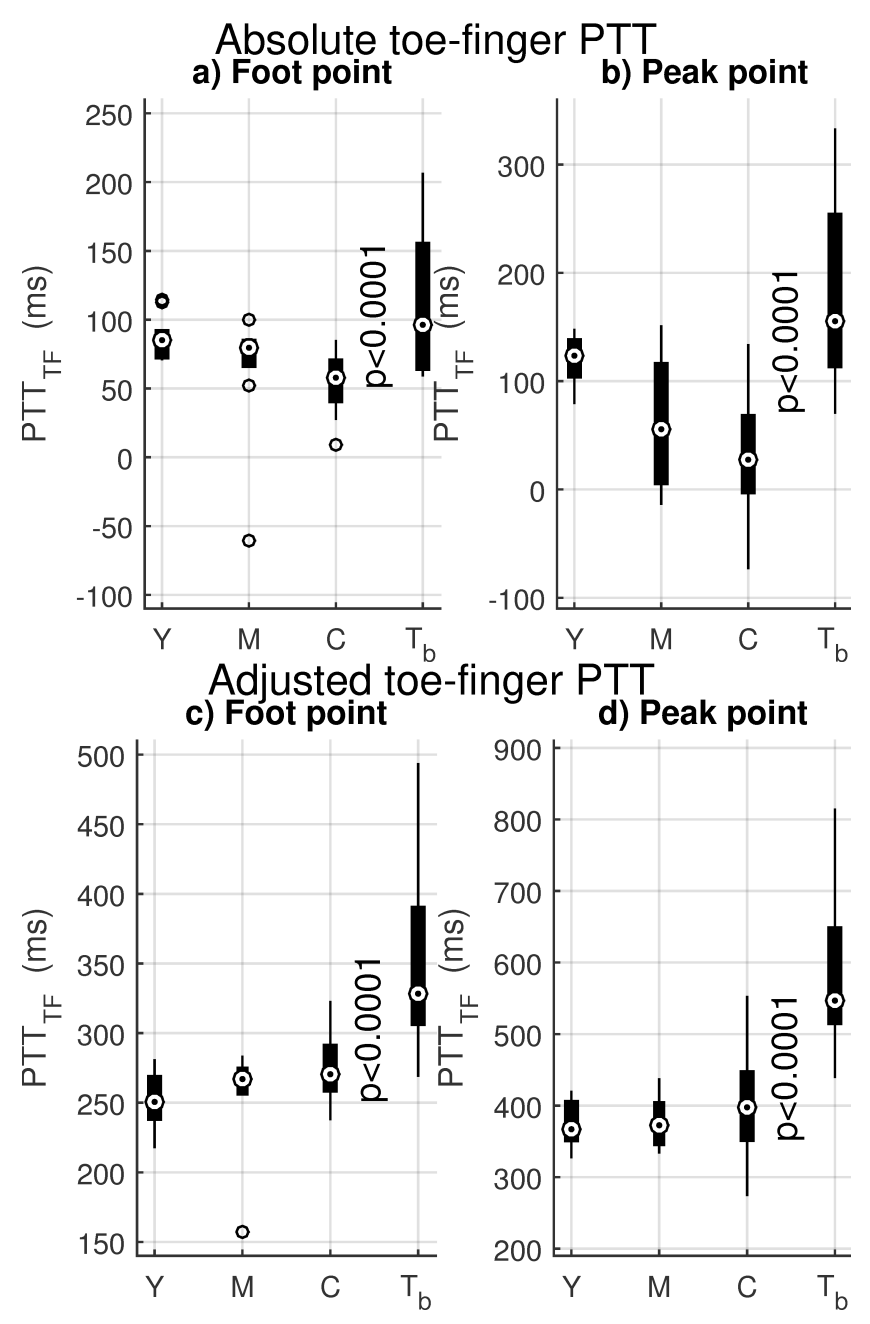

Figure 5. Distributions of the absolute (panels a-b) and adjusted (panels c-d) toe-finger PTTs for each group. $\mathrm{T}_{\mathrm{b}}=$ treated lower limb before the treatment, $\mathrm{C}=$ at least 65 -year-old control subject, $\mathrm{M}=$ middle-aged subjects, $\mathrm{Y}=$ young subjects. P-values are for 2-tailed Mann-Whitney U-test betweeen $\mathrm{T}_{\mathrm{b}}$ and $\mathrm{C}$.

Table IV

FREE-MARGINAL MULTIRATER $\kappa$ VALUES FOR BETWEEN PTTS AND REFERENCE VALUES (ABI AND TOE PRESSURE).

\begin{tabular}{l|lc|cc}
\hline \hline Reference & \multicolumn{2}{|c|}{ Lower-limb ECG-PTT } & \multicolumn{2}{c}{ Toe-finger PTT } \\
point & ABI & Toe pressure & ABI & Toe pressure \\
\hline Foot & 0.55 & 0.33 & 0.90 & 0.80 \\
Peak & 0.91 & 0.71 & 0.81 & 0.70 \\
1st d. & 0.91 & 0.71 & 1.00 & 0.90 \\
2nd d. & 0.55 & 0.33 & 0.90 & 0.80 \\
Int. tan. & 0.73 & 0.52 & 1.00 & 0.90 \\
\hline Toe pressure & 0.75 & & \\
1st d.: 1st derivative; 2nd d.: 2nd derivative; int. tan.: Intersecting tange
\end{tabular}

agreement between $\mathrm{ABI}$ than the mutual $\kappa$-agreement $(0.75)$ between the reference, toe pressures and ABI.

\section{DISCUSSION}

The main finding of this study is that the lower limb PTTs have a U-shaped dependence on arterial condition which is affected by aging, independently of the method how the PTTs are defined. Our hypothesis based on the results is that the lower limb PTTs decrease with the age as a result of lost compliance i.e. arterial stiffening since the PW velocity 


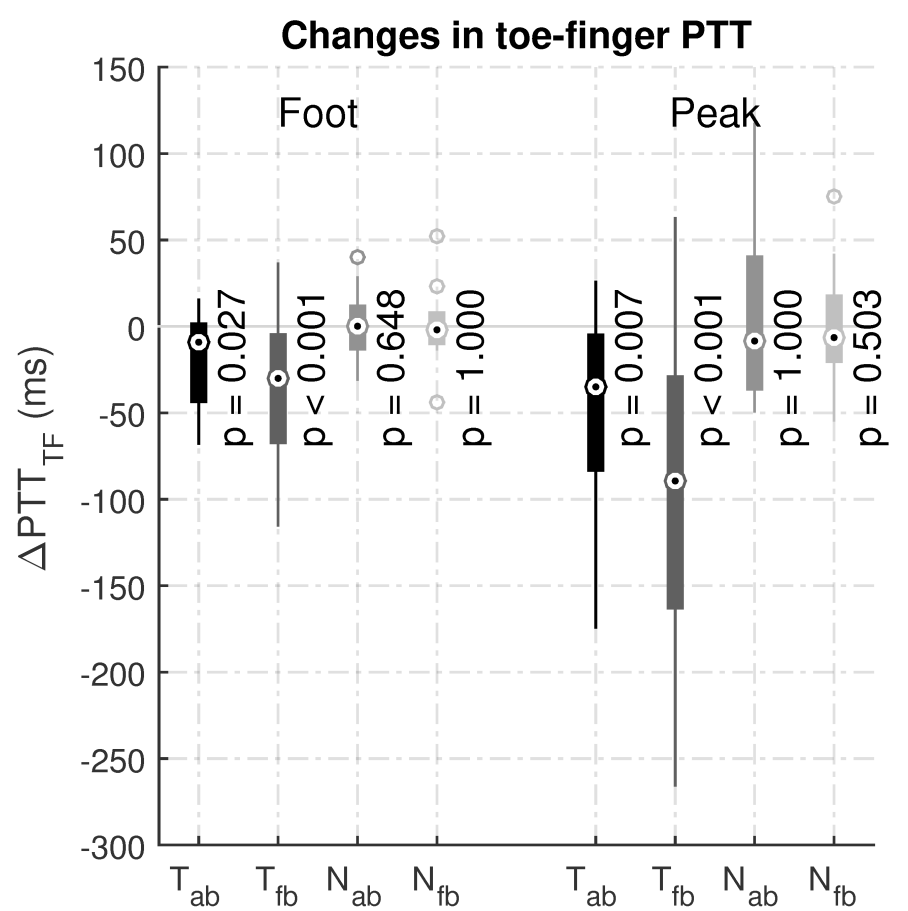

Figure 6. Changes in toe-finger PTTs. Labels $\mathrm{T}$ and $\mathrm{N}$ refer treated lower limb and non-treated lower limb, respectively. Subscript ab refers to difference between pre-treatment ( $b=$ before) and immediate post-treatment $(a=a f t e r)$ conditions, and subscript fb refers to difference between pre-treatment condition and follow-up visit.

(PWV) increases. However, lower limb PTTs increase with the existence of PAD lesions as the flow resistance increases and because the blood pressure in the lower limb decreases. The PTTs decrease as a result of the PTA once the PAD lesion is successfully dilated and vascular remodellation has begun. Change in the PTTs between immediate post-treatment and follow-up phases suggests that the remodellation initiated by the treatment continues after it.

Based on the presented results, the peak-point based PTTs have the best overall performance in finding the markers of PAD: The separation between the distributions of the control subjects and PAD-patients' pre-treatment state of the treated lower limb is the greatest (Figs. 2 and 5), and the differences between the pre- and post-treatment PTTs are consistent in the treated lower limbs and zero-centered in non-treated lower limbs (Figs. 4 and 6). The agreement with the reference values is also good (Table IV).

The behaviour of the PTTs in the upper limbs is different from that in the lower limbs. As the upper limbs are less prone to stenoses or occlusions, the PTTs of the upper limbs tend to decrease with age except the peak point based PTT (Table III). As the central and peripheral arterial stiffening increases the PWV, the beginning of the rising edge of the PW arrives earlier to a measurement point in upper limb. The different behaviour of the peak-point based upper limb PTT may provide information on other vascular diseases or information on vascular health for different applications, but requires further studies. We assume that the peak-point of the upper limb PW is delayed in stiffened arteries, since the reflected waves from the impedance discontinuities of the arterial tree arrive earlier and overlap with the heartbeat induced percussion wave. This causes delayed timing of the peak compared with the distinct waves. The overlapping increases also the systolic blood pressure compared with the healthy elastic arteries, in which the percussion wave and the first reflected wave are often clearly distinct. In our results, the differences in the upper limb PTTs between the control group and the pre-treatment state of the patients are clearly less obvious and less significant than the differences in the lower limbs.

The presented results are in line with earlier studies: Yokoyama et al. [15] have reported increase in brachial-ankle PWV in diabetic patients after undergoing a PTA. There are also studies that present results for increased PTT in the lower limb having PAD lesions [6]-[8]. In addition, Nitzan et al. [4] found negative correlation between age and foot-point based ECG-PTT and toe-finger PTT, and Allen et al. [5] found negative correlation between age and foot-point based upper and lower limb ECG-PTT.

The prevalence of PAD is higher in elderly people. If the results of the present and earlier studies [4]-[8] are combined, the lower limb PTTs as such cannot be utilized to characterize the status of vasculature since the age decreases the PTT whereas PAD increases it. According to [4], [5], age is a dominating factor affecting the PTTs compared with height and blood pressure. Our results also support a strong PTT dependence on age and PAD. However, the presented compensation coefficients are not completely validated as we did not have a separate validation group of young and middleaged subjects. Reference values for the age and systolic blood pressure dependence were found from literature only for the foot-point based PTTs (Table III). Our values for the toe-based ECG-PTT have the same order of magnitude as the values based on univariate regression and presented in [5], but the deviations are higher for coefficients found for the other footpoint based PTTs..

The most significant limitation for the generalization of the results is the lack of height compensation. Related studies have reported height-dependency coefficients of $1.1 \mathrm{~ms} / \mathrm{cm}$ [5] and $1.03 \mathrm{~ms} / \mathrm{cm}$ [4] for foot-point and toe-based ECGPTT, $0.59 \mathrm{~ms} / \mathrm{cm}$ [4] for foot-point based finger-toe PTT, and $0.7 \mathrm{~ms} / \mathrm{cm} \mathrm{[4]} \mathrm{for} \mathrm{foot-point} \mathrm{and} \mathrm{index} \mathrm{finger} \mathrm{based}$ ECG-PTT. The heights of $165 \mathrm{~cm}-185 \mathrm{~cm}$ are expected to cause a variation in maximum of roughly $22 \mathrm{~ms}$. The median height of the PAD-patients is approximately $10 \mathrm{~cm}$ shorter than the median height of the younger groups. If this could be taken into account, the PAD-patients should be even more distinct group from the younger subjects. However, the height distributions of younger groups ( $\mathrm{Y}$ and $\mathrm{M}$ ) as well as PADpatients and control subjects were matched (Table I), making both pairwise comparisons of between equally-height groups reasonable. Even though we were not able to take into account the height-dependence with our data, we can quantify that the lower limb PTTs are affected by the PAD lesions since there are clear differences at group level in the PTT values between the pre-treatment and post-treatment (immediate posttreatment and follow-up visit) conditions in the treated lower limb. These differences are less significant or negligible in 
non-treated lower limb and in upper limb.

All PAD-patients except one met a criterion ABI $<0.9$ which limits the generalization of the results to the PAD patients having $\mathrm{ABI}>1.3$. Even though the PTT distributions of control subjects (C) and pre-treatment values of the treated limb $\left(T_{b}\right)$ differ significantly, we observed that the distributions of pre-treatment PTTs of treated $\left(\mathrm{T}_{\mathrm{b}}\right)$ and non-treated limb are strongly overlapping (see Supplementary material). The most probable reason to this is that the lesions of PAD are often bilaterally present, which is the case also in this study: 68 $\%$ of the patients had abnormal ABI-reading in non-treated lower limb at least in one of the two measurements. There is a limitation also related to a group of control subjects: the exclusion of PAD was based on ABI measurement and a careful risk factor survey, nonetheless the ABI has been reported to have limitations such as varying sensitivity and specificity from study to study as well as difficulties in interpretations of mediasclerotic patients' results. [1], [3].

\section{Conclusions}

In this study, we compared both upper and lower limb PTTs based on different reference points and measured from patients undergoing PTA for the SFA in immediate pre- and posttreatment conditions as well as the situation in the follow-up visit with the PTTs measured from younger, middle-aged and elderly control subjects. The results showed that the lower limb PTTs decrease with age, but increase with the existence of stenoses caused by PAD. The vascular remodellation initiated by the PAD is seen as a decrease in post-treatment PTT-values. The presented results are in line with the results found in the literature in terms of age dependence and PAD diagnosis. The changes in many PTT-measures as a result of the PTA have better agreement with the changes of the clinical reference values ABI and toe pressure than the mutual agreement of these reference values. Based on the presented results of different PTT-measures, the lower limb PTTs calculated using the peak point of the PW have the best overall performance in finding PAD lesions. The arterial diseases are a complex continuum of different factors affecting diagnostic tests. Rapid and costeffective PPG-measurement and automated analysis of (lowerlimb) PWs could be an additional tool in daily clinical practice or screening studies of PAD, as the current methods (ABI, TBI, toe pressures) have limitations in their performance. The data combined from different measurement modalities of cardiovascular system could provide better understanding about patient's vascular health. Further studies are required to develop the method of PW-measurements into an established examination modality.

\section{ACKNOWLEDGMENT}

The authors would like to thank all the volunteer test subjects for their valuable contribution. The personnel in the Policlinics of Vascular Surgery at Tampere University Hospital and Oulu University Hospital are acknowledged for their contribution in collecting the reference values.

The work was funded by the Doctoral Programme of the President of Tampere University of Technology, Business
Finland as a part of project VitalSens (decision ID 40103/14), Academy of Finland as part of project Vascular Biomechanics Assessment using printed soft electronics and advanced signal processing (VBA) (decisions 310617 and 310618) and grants from Finnish Cultural Foundation/Pirkanmaa Regional Fund, Tekniikan edistämissäätiö, and Emil Aaltonen Foundation.

\section{REFERENCES}

[1] D. Xu, J. Li, L. Zou, Y. Xu, D. Hu, S. L. Pagoto, and Y. Ma, "Sensitivity and specificity of the ankle-brachial index to diagnose peripheral artery disease: a structured review," Vascular Medicine, vol. 15, no. 5, pp. 361-369, 2010.

[2] M. T. Romanos, A. Raspovic, and B. M. Perrin, "The reliability of toe systolic pressure and the toe brachial index in patients with diabetes," Journal of foot and ankle research, vol. 3, no. 1, p. 31, 2010.

[3] S. Alatab, H. Fakhrzadeh, F. Sharifi, A. Mostashfi, M. Mirarefin, Z. Badamchizadeh, and Y. Tagalizadehkhoob, "Impact of hypertension on various markers of subclinical atherosclerosis in early type 2 diabetes," Journal of Diabetes \& Metabolic Disorders, vol. 13, no. 1, p. 1, 2014.

[4] M. Nitzan, B. Khanokh, and Y. Slovik, "The difference in pulse transit time to the toe and finger measured by photoplethysmography," Physiological measurement, vol. 23, no. 1, p. 85, 2002.

[5] J. Allen and A. Murray, "Age-related changes in peripheral pulse timing characteristics at the ears, fingers and toes," Journal of human hypertension, vol. 16, no. 10, p. 711, 2002.

[6] R. Erts, J. Spigulis, I. Kukulis, and M. Ozols, "Bilateral photoplethysmography studies of the leg arterial stenosis," Physiological measurement, vol. 26, no. 5, p. 865, 2005.

[7] J. Allen, C. P. Oates, T. A. Lees, and A. Murray, "Photoplethysmography detection of lower limb peripheral arterial occlusive disease: a comparison of pulse timing, amplitude and shape characteristics," Physiological measurement, vol. 26, no. 5, p. 811, 2005.

[8] J. Allen, K. Overbeck, A. F. Nath, A. Murray, and G. Stansby, "A prospective comparison of bilateral photoplethysmography versus the ankle-brachial pressure index for detecting and quantifying lower limb peripheral arterial disease," Journal of vascular surgery, vol. 47, no. 4, pp. 794-802, 2008.

[9] M. Peltokangas, A. Vehkaoja, J. Verho, M. Huotari, J. Röning, and J. Lekkala, "Monitoring arterial pulse waves with synchronous body sensor network," Biomedical and Health Informatics, IEEE Journal of, vol. 18, no. 6, pp. 1781-1787, Nov 2014.

[10] G. Oetken, T. Parks, and H. Schuessler, "A computer program for digital interpolator design," Digital Signal Processing Committee (ed) Programs for Digital Signal Processing. IEEE Press, New York, vol. 8, pp. 8.11-8.1-6, 1979.

[11] M. Peltokangas, A. Vehkaoja, J. Verho, V. M. Mattila, P. Romsi, J. Lekkala, and N. Oksala, "Age dependence of arterial pulse wave parameters extracted from dynamic blood pressure and blood volume pulse waves," IEEE journal of biomedical and health informatics, vol. 21, no. 1, pp. 142-149, 2017.

[12] S. C. Millasseau, R. P. Kelly, J. M. Ritter, and P. J. Chowienczyk, "The vascular impact of aging and vasoactive drugs: comparison of two digital volume pulse measurements," American journal of hypertension, vol. 16, no. 6, pp. 467-472, 2003.

[13] Y. C. Chiu, P. W. Arand, S. G. Shroff, T. Feldman, and J. D. Carroll, "Determination of pulse wave velocities with computerized algorithms," American heart journal, vol. 121, no. 5, pp. 1460-1470, 1991.

[14] J. Randolph, "Free-marginal multirater kappa (multirater $\kappa$ free): An alternative to fleiss' fixed-marginal multirater kappa," in Presented at the Joensuu Learning and Instruction Symposium, vol. 2005, 2005.

[15] H. Yokoyama, T. Shoji, E. Kimoto, K. Shinohara, S. Tanaka, H. Koyama, M. Emoto, and Y. Nishizawa, "Pulse wave velocity in lower-limb arteries among diabetic patients with peripheral arterial disease," Journal of atherosclerosis and thrombosis, vol. 10, no. 4, pp. 253-258, 2003. 